\title{
La técnica de grupos focales como método para el desarrollo de la competencia comunicativa intercultural
}

\author{
Lluís Valls Campà
}

Palabras clave: Competencia comunicativa intercultural, didáctica, ELE, etnografía, grupos focales.

\section{Introducción}

A pesar de los avances teóricos y metodológicos en la enseñanza/aprendizaje de la comunicación intercultural, actualmente la competencia intercultural está poco integrada en el aula de lengua extranjera (LE). Uno de los pocos estudios existentes sobre la situación real de la enseñanza de la comunicación intercultural en el aula de LE en Europa (Sercu et al., 2005) concluye que la mayoría de los profesores no incorpora una orientación intercultural a sus clases, ya que en ellas no está presente la adquisición de conocimientos, actitudes y habilidades interculturales. En su lugar, los profesores se centran en la adquisición de la competencia comunicativa, predominando la subcompetencia lingüística, y la presencia de la cultura en el aula no tiene más que el objetivo de conseguir cierta familiaridad de los alumnos con la vida cotidiana en la sociedad de la lengua meta.

Sin embargo, según el mismo estudio, muchos de los profesores son favorables a la enseñanza/aprendizaje de la competencia intercultural y desean integrarla más en el aula, pero se encuentran limitados, además de por la orientación del currículo de sus centros hacia la competencia lingüística, por su propia falta de formación intercultural. De forma parecida, el Plan Curricular del Instituto Cervantes se hace eco de que "los profesores, en 
general, no disponen de la documentación necesaria o de la metodología adecuada que les ayude a determinar qué datos sociales, culturales y sociolingüísticos buscar, y cómo y dónde encontrarlos" (Instituto Cervantes, 2007: 39-40).

Con la intención de proporcionar un nuevo método para el desarrollo de la competencia comunicativa intercultural de los alumnos de ELE, que sea aplicable en clases de español realizadas en el extranjero y, por lo tanto, con difícil acceso a elementos culturales de la sociedad de la lengua meta, en este artículo presentamos la adaptación didáctica de la técnica de investigación social denominada grupos focales.

\section{El enfoque intercultural}

\subsection{La competencia comunicativa intercultural}

El enfoque intercultural en la enseñanza de LE se ha ido desarrollando desde los años 80, debido a la insatisfacción con el enfoque comunicativo de autores que consideran que no da el suficiente protagonismo a la enseñanza/aprendizaje de los conocimientos y la interacción intercultural (Byram y Esarte-Sarries, 1991; Byram, 1997; Byram, Nichols y Stevens 2001; Byram, Nichols y Stevens, 2003; Corbett, 2003). Ello es debido, por un lado, a que el método comunicativo se ha centrado en el desarrollo de las funciones comunicativas, a partir del intercambio de información basado en el vacío de información de las actividades didácticas, olvidando las funciones sociales de la lengua; y por otro lado, a que los autores de materiales didácticos tampoco han sabido cómo introducir actividades interculturales en los mismos (Corbett 2003). Byram (1997) ha establecido de forma clara las carencias del enfoque comunicativo (EC):

1. La corrección lingüística y la adecuación al contexto del EC no son suficientes para conseguir una comunicación efectiva, sino que además hace falta la empatía del hablante para ponerse en la situación de su interlocutor, considerando cómo este interpreta el mensaje según su marco de referencia cultural.

2. El EC ignora que para conseguir una comunicación efectiva es 
necesario el establecimiento de una actitud positiva entre los interlocutores para relacionarse, entenderse y superar los conflictos y malentendidos que puedan producirse por las diferencias de valores y formas de interacción de cada cultura.

3. La persona que aprende una nueva lengua posee ya su propia competencia lingüística y cultural y su propia identidad social, adquiridas en el proceso de socialización, las cuales están también presentes cuando se interacciona con personas de otras culturas, hecho que es ignorado por el EC.

4. El EC toma por modelo a imitar la competencia lingüística y cultural del hablante nativo con con buen nivel educativo, lo cual, además de no ser una meta realista, lleva a considerar como modelo la cultura de los grupos dominantes en la sociedad de la LE e ignorar la heterogeneidad de la misma, y sitúa al hablante extranjero en una situación de subordinación al hablante nativo, el cual "siempre tiene la razón" sobre cómo debe hablar y comportarse su interlocutor.

Para corregir estas limitaciones, el enfoque intercultural propone como objetivo de la enseñanza/aprendizaje de la LE, no solo el desarrollo de la competencia comunicativa, sino el desarrollo de la competencia comunicativa intercultural (CCI), la cual puede ser definida como "la habilidad de comprender y relacionarse con gente de otros países” (Byram, 1997: 5).

\subsection{Componentes de la competencia comunicativa intercultural}

Según el modelo intercultural propuesto por Byram (1997), la competencia comunicativa intercultural está compuesta por las competencias de la lengua (lingüística, socioligüística y discursiva), y por la competencia intercultural. Para que el alumno consiga establecer una comunicación eficiente con hablantes de otros países, además de conocimientos culturales sobre esos países, necesita adquirir una serie de actitudes y habilidades para descubrir y entender los elementos culturales y para su análisis crítico. Así, la competencia intercultural en el modelo de Byram está formada por cinco componentes: actitudes para apreciar los valores, creencias y conductas de los 
otros y relativizar los propios (saber ser); conocimientos culturales sobre uno mismo y sobre el otro, y de la interacción intercultural (saberes); habilidades para interpretar y relacionar elementos culturales (saber comprender); habilidades para descubrir y/o interactuar (saber aprender/hacer); y una conciencia cultural crítica (saber relacionarse). ${ }^{1)}$

\subsection{El alumno como etnógrafo}

Desde el enfoque intercultural se pone el énfasis en que el alumno de lengua extranjera desempeñe un rol activo en la adquisición de los conocimientos culturales, buscando él mismo datos sobre los comportamientos culturales a través de la observación y la realización de entrevistas, y reflexionando sobre los datos obtenidos para describir esos comportamientos, con lo cual el alumno puede adquirir no solo conocimientos sino también habilidades interculturales. Por ello, se ha propuesto que el aprendiente de LE se convierta en pseudo-etnógrafo; es decir, que el alumno "aprenda la lengua y la cultura como un todo con el objetivo de describir y entender a la gente en cuestión" (Byram y Esarte-Sarries, 1991, p. 11) ${ }^{2}$. Así, el objetivo del aprendizaje de la lengua extranjera se convierte en conocer y comprender otras culturas, y en las clases se adquieren conocimientos lingüísticos y culturales simultáneamente.

Con el método etnográfico los alumnos de LE realizan una investigación etnográfica sobre un determinado grupo social perteneciente a la lengua meta. Este tipo de actividad es denominado proyecto a gran escala ya que, entre la fase de aprendizaje de la metodología etnográfica, la fase de preparación de la investigación, la realización de la misma y la preparación del informe final, pueden transcurrir desde varias semanas hasta varios años. Una investigación etnográfica consta de siete etapas (Corbett, 2003):

1. Elección del grupo y el tema objeto de estudio.

2. Selección de los informantes.

3. Revisión bibliográfica.

4. Observación y entrevistas a los informantes.

5. Análisis de los datos y generación de hipótesis culturales que 
expliquen el comportamiento del grupo en cuestión.

6. Reflexión sobre los marcos de referencia con los que se han generado las hipótesis, para conocer sus limitaciones y mejorarlas.

7. Elaboración del informe, presentación y discusión en clase.

Los proyectos a gran escala tienen la ventaja de que promueven la adquisición de todos los componentes de la CCI. Tanto en la etapa de preparación del proyecto como durante el desarrollo de la investigación, los aprendientes incrementan sus conocimientos sobre el grupo social investigado y su cultura; al preparar y realizar las observaciones y entrevistas, los aprendientes adquieren las habilidades de descubrimiento e interacción, y al analizar los resultados de las mismas adquieren las habilidades de interpretación y relación; tanto al interpretar los resultados como al reflexionar sobre los marcos de referencia con los que se interpretan los resultados, los aprendientes adquieren actitudes de distanciamiento de sus propios marcos de referencia culturales y actitudes de empatía y de curiosidad, buscando la explicación de las conductas observadas en elementos culturales del grupo social en cuestión. Además, durante todo proceso los aprendientes necesitan leer, hablar y escribir en la lengua meta, con lo cual se adquieren también las competencias lingüística, sociolingüística y discursiva.

Por otro lado, los proyectos etnográficos a gran escala tienen el inconveniente de que puede haber dificultades para acceder a grupos de la sociedad de la lengua meta, sobre todo si los alumnos se encuentran en su país de origen (Corbett, 2003).

\section{La investigación de grupos focales}

\subsection{Definición y características de la técnica}

Hemos visto que los proyectos etnográficos a gran escala incluyen la técnica de observación sistemática y la técnica de entrevista individual como métodos para que el alumno obtenga datos de la cultura y adquiera habilidades y actitudes interculturales. En este apartado presentamos una técnica diferente, que incluye aspectos de la observación sistemática y aspectos de la entrevista, a la vez que posee otras características propias. 
La técnica de grupos focales (focus groups) o grupos de discusión, es una técnica de tipo cualitativo muy utilizada en estudios de sociología, psicología $y$, sobre todo, en estudios de marketing. Consiste en la organización de pequeños grupos de discusión sobre un tema determinado que, dirigidos por las preguntas del moderador, proporcionan datos que permiten analizar las opiniones, conductas y sentimientos de un determinado grupo social. Esta técnica permite también analizar la interacción entre los miembros del grupo durante la discusión (Morgan, 1997; Krueger y Casey, 2000; Stewart, et al,. 2007).

La primera característica de la técnica de grupos focales es la denominada focalización. La focalización consiste en que la discusión versa sobre un tema concreto previamente definido por el investigador, con la intención de recoger datos que le permitan dar respuesta al problema de investigación planteado. Además, los participantes en la discusión comparten algunas características que los relacionan con el tema de discusión. Así, por ejemplo, en sociología y psicología social hay investigaciones que se han focalizado en una clase social o profesional para conocer su estilo de vida, organizando grupos de discusión formados por personas de la misma profesión. También, en marketing, frecuentemente se organizan grupos focales formados por consumidores de un determinado tipo de producto, discutiendo sobre sus patrones de consumo, o valorando un determinado producto o anuncio publicitario (Stewart et al., 2007).

La segunda característica de la técnica es que, al ser una discusión en grupo, se pueden observar las interacciones entre los miembros del grupo: "permite observaciones de cómo y por qué los individuos aceptan o rechazan las ideas de los demás" (Stewart et al., 2007: 10).

La tercera característica es la denominada profundidad de los datos recogidos. Con la técnica de grupos focales no se pretende únicamente que los participantes expliquen su conducta, sino que además se pretende conocer sus motivaciones y sentimientos en relación al tema de discusión (Stewart et al., 2007).

Finalmente, se considera que esta técnica tiene un valor humanístico, ya que los participantes en el grupo focal tienen la oportunidad de expresar 
libremente sus opiniones sobre un tema que les afecta. Además, como la recogida de datos es en forma de discusión en grupo, se promueve entre los participantes la empatía y actitudes positivas respecto de la interacción con otras personas, como escuchar y aceptar otras opiniones(Stewart et al., 2007).

La técnica de grupos focales tiene similitudes y diferencias, ventajas y desventajas, en comparación con las dos técnicas que se utilizan habitualmente en el método etnográfico (Tabla 1). En cuanto a la observación sistemática, tanto en esta técnica como en la técnica de grupos focales se observan las interacciones entre los individuos del grupo que se estudia. Sin embargo, mientras que en la primera el investigador se limita a observar conductas que se producen de forma espontánea en el lugar y el momento de la observación, en los grupos focales las interacciones son estimuladas y dirigidas por las preguntas del investigador. Por otro lado, en la técnica de grupos focales, al hablarse directamente con los participantes, se pueden conocer sus experiencias pasadas y conductas no observables, tales como las opiniones y los sentimientos. Estas diferencias implican que la técnica de grupos focales tiene la desventaja de que la situación no es natural para los

Tabla 1: Comparación de técnicas de recogida de datos. Elaboración propia a partir de Morgan, 1997 y Stewart et al., 2007.

\begin{tabular}{|l|l|l|l|}
\hline & $\begin{array}{l}\text { Observación } \\
\text { sistemática }\end{array}$ & Grupos focales & $\begin{array}{l}\text { Entrevista } \\
\text { individual }\end{array}$ \\
\hline $\begin{array}{l}\text { Control de } \\
\text { la situación }\end{array}$ & $\begin{array}{l}\text { Ausencia de } \\
\text { control }\end{array}$ & Control medio & Control elevado \\
\hline $\begin{array}{l}\text { Número de } \\
\text { sujetos }\end{array}$ & $\begin{array}{l}\text { Variable y sin } \\
\text { control }\end{array}$ & Grupo pequeño & Un solo sujeto \\
\hline $\begin{array}{l}\text { Contexto } \\
\text { espacial y } \\
\text { temporal }\end{array}$ & $\begin{array}{l}\text { El lugar y el } \\
\text { momento de la } \\
\text { observación }\end{array}$ & $\begin{array}{l}\text { El lugar y el momento } \\
\text { de la observación y } \\
\text { experiencias anteriores }\end{array}$ & $\begin{array}{l}\text { El lugar y el } \\
\text { momento de la } \\
\text { observación y } \\
\text { experiencias } \\
\text { anteriores }\end{array}$ \\
\hline $\begin{array}{l}\text { Tipo de } \\
\text { datos } \\
\text { recogidos }\end{array}$ & $\begin{array}{l}\text { Conductas } \\
\text { observables }\end{array}$ & $\begin{array}{l}\text { Conductas observables } \\
\text { y conductas no } \\
\text { observables }\end{array}$ & $\begin{array}{l}\text { Básicamente } \\
\text { conductas no } \\
\text { observables }\end{array}$ \\
\hline
\end{tabular}


participantes, y la variedad de conductas observables es menor que en la observación sistemática. Por otra parte, tiene la ventaja de que en un espacio limitado de tiempo pueden obtenerse muchos datos sobre el tema de estudio (Morgan, 1997; Stewart et al., 2007).

En cuanto a la técnica de entrevista individual, tanto esta técnica como la de grupos focales permiten al entrevistador dirigir la entrevista con sus preguntas y seleccionar los temas que se tratan en la misma. Pero, debido al menor control por parte del moderador y a la pluralidad de participantes en el grupo, la técnica de grupos focales tiene las desventajas de que permite hacer menos preguntas a cada sujeto en particular y que la dirección de la discusión resulta más difícil para el moderador. Por otro lado, tiene una serie de ventajas: permite obtener datos de diferentes individuos a la vez, comparar sus respuestas y analizar las reacciones mutuas de los participantes, y las respuestas de unos pueden aportar nuevas ideas para la discusión y estimular la participación de los demás (Morgan, 1997; Stewart et al., 2007).

\subsection{Fases en una investigación con grupos focales}

Stewart et al. (2007) describen ocho fases en una investigación realizada con la técnica de grupos focales:

1. Definición del problema de investigación. En primer lugar se fija el tema de interés y, dentro del mismo, se define el problema concreto a investigar y el tipo de datos que se requieren para dar respuesta a ese problema. Es necesario que el problema esté bien definido, especificando la pregunta a la que se quiere encontrar respuesta con la investigación.

2. Definición del grupo de estudio. Los miembros del grupo objeto de estudio tienen alguna característica en común que los relaciona con el problema de investigación, por ejemplo, haber vivido una determinada experiencia, formar parte de la misma generación, ser consumidores de un determinado tipo de producto, etc.

3. Selección del moderador. El moderador debe tener ciertos conocimientos sobre el área temática sobre la que se va a discutir, y 
poseer formación sobre moderación de grupos de discusión.

4. Elaboración de la guía de discusión. Esta es una de las fases más importantes en la investigación. La guía está formada por las preguntas que el moderador va a realizar durante la discusión en grupo y el orden en que piensa preguntarlas. Ya que el objetivo es establecer una hoja de ruta para la discusión en grupo, tanto las preguntas como el orden de las mismas no es inamovible y la guía puede ser modificada durante la discusión.

5. Reclutamiento de los participantes. Se reclutan los sujetos que van a participar en el grupo de discusión. Se considera que el número de participantes en los grupos de discusión debe ser de entre seis y doce personas (Morgan, 1997; Stewart et al., 2007).

6. Realización de la discusión en grupo. El grupo discute entre 60 y 90 minutos sobre el tema propuesto por el moderador y guiado por sus preguntas. La discusión es observada (tanto en su contenido como en su forma), por el mismo moderador y por observadores, y se toman registros de la misma, ya sean notas, vídeo o audio. Para que la discusión tenga éxito, su desarrollo debe seguir cuatro principios (Morgan, 1997): amplitud, especificidad, profundidad y contexto personal. La amplitud se refiere a que se deben cubrir todos los temas que se consideren relevantes para dar respuesta al problema de investigación, aunque no hayan estado previstos en la guía de la entrevista. Por ello, los temas introducidos por los participantes durante la discusión pueden ser una fuente de nuevas preguntas. En cuanto a la especificidad, las respuestas de los participantes deben explicar sus propias experiencias y pensamientos. Las respuestas deben ser profundas en el sentido de que deben expresar las motivaciones y las emociones de los participantes respecto de los temas tratados. Finalmente, el contexto personal se refiere a que las preguntas deben indagar sobre las experiencias y situaciones personales que llevan al sujeto a elaborar una determinada respuesta; se debe intentar contestar a la pregunta: “¿por qué este sujeto contesta de esta manera a esta cuestión?” (Morgan, 1997, p. 46). 
7. Análisis e interpretación de los datos. Generalmente el análisis de datos es de tipo cualitativo y existen diferentes niveles de análisis según su grado de sofisticación estadística. El nivel de análisis más básico consiste en la realización de un resumen de la discusión y la elaboración de las conclusiones a partir del mismo. No se trata de realizar generalizaciones a partir de las respuestas de los participantes, sino de comprender los motivos de dichas respuestas.

8. Elaboración del informe. Debe contener el resumen de la discusión y las conclusiones. Estas deben dar respuesta al problema de investigación planteado.

\subsection{Preparación de la guía de discusión}

Tanto al elaborar la guía, como al ejecutarla durante el desarrollo de la discusión, deben tenerse en cuenta una serie de principios para formular preguntas que estimulen una discusión eficaz. En primer lugar, las preguntas deben estar formuladas de forma natural como en una conversación; deben estar formuladas con palabras que usarían los participantes en la discusión al hablar sobre ese tema, evitándose tecnicismos y expresiones a las que no están habituados; deben ser fáciles de decir oralmente, para que puedan ser comprendidas con facilidad; deben ser claras, no mezclando diferentes preguntas ni induciendo a la confusión; deben ser cortas; generalmente deben implicar respuestas abiertas, evitándose las respuestas del tipo "sî" o "no"; deben ser fáciles de contestar, no requiriendo para ello pensar en exceso; deben ser respetuosas y no incluir presuposiciones sobre las conductas de los participantes (Krueger y Casey, 2000; Stewart et al., 2007).

En segundo lugar, otro aspecto importante que el moderador debe decidir es el orden de las preguntas según su grado de amplitud o concreción. La forma más habitual es ordenarlas en forma de "embudo", es decir empezar con las preguntas más generales, que permiten conocer la opinión general de los diferentes miembros respecto al tema tratado, para luego preguntar sobre aspectos determinados del tema sobre los que interesa conocer más profundamente (Krueger y Casey, 2000; Stewart et al., 2007). Sin embargo, el orden inverso ("embudo invertido") también se utiliza para facilitar la 
participación de los sujetos, planteando cuestiones concretas de respuesta fácil al principio y promoviendo una discusión más libre con preguntas posteriores más generales (Stewart et al., 2007).

Además, el moderador debe elegir qué tipo de preguntas realiza en cada momento según su función dentro del desarrollo de la discusión. La sesión del grupo focal se inicia con preguntas de apertura, para romper el hielo, y preguntas introductorias, para presentar el tema e iniciar la discusión. Luego siguen las cuestiones de transición, con la función de avanzar hacia las preguntas clave. Las preguntas de transición pueden ser de diversos tipos: indicativas (dan instrucciones a los participantes para realizar alguna actividad), tentativas (tantean un determinado tema), directivas (vuelven la discusión hacia el tema principal cuando se ha desviado del mismo), obtusas (dirigen la discusión hacia un nivel más general cuando la sensibilidad del tema crea incomodidad en los sujetos), y tamién se puede utilizar el silencio, el cual permite la intervención espontánea de los participantes. Las preguntas clave deben estar bien preparadas, y el moderador debe indagar tanto como crea necesario para conseguir respuestas concretas y explorar a fondo el tema. Para terminar la discusión se realizan preguntas finales para reflexionar sobre lo ya dicho y comprobar que no haya confusiones (Krueger y Casey, 2000; Stewart et al., 2007).

Finalmente, se debe determinar qué tipo de acciones se va a pedir que realicen los miembros del grupo. Las preguntas, además de requerir una respuesta verbal sobre las opiniones y experiencias de los sujetos, pueden solicitar que estos realicen diferentes actividades, como dibujar, elegir entre varias fotos, elaborar listas de palabras, etc. (Tabla 2). Estas actividades, por un lado, proporcionan de forma directa datos sobre las opiniones de los sujetos; por otro lado, algunas de estas actividades implican técnicas proyectivas, buscando conocer indirectamente las actitudes y los sentimientos de los que el sujeto no es consciente. ${ }^{4}$ Estas actividades también sirven como base para la comparación de las opiniones de los participantes y para estimular la discusión (Krueger y Casey, 2000; Stewart et al., 2007). 
Tabla 2: Tipos de preguntas según la actividad. Elaboración propia a partir de Krueger y Casey, 2000 y Stewart et al. 2007.

\begin{tabular}{|l|l|}
\hline $\begin{array}{l}\text { Hacer listas, } \\
\text { ordenar, } \\
\text { elegir }\end{array}$ & $\begin{array}{l}\text { Se hacen listas de preferencias, se ordenan objetos según un } \\
\text { criterio determinado, se elige entre opciones, y se discute } \\
\text { sobre los motivos de la elección. }\end{array}$ \\
\hline $\begin{array}{l}\text { Comentar } \\
\text { fotos }\end{array}$ & $\begin{array}{l}\text { Se atribuyen conductas y características a personajes } \\
\text { presentados en fotos, se discute sobre los motivos de la } \\
\text { atribución. }\end{array}$ \\
\hline Dibujar & $\begin{array}{l}\text { Se realiza un dibujo que exprese una determinada conducta o } \\
\text { actitud, luego se compara con los otros participantes y se } \\
\text { discute sobre los motivos de los dibujos. }\end{array}$ \\
\hline $\begin{array}{l}\text { Imaginar } \\
\text { previas }\end{array}$ & $\begin{array}{l}\text { Se imagina cómo podrían ser las cosas en un mundo ideal } \\
\text { (por ejemplo, la solución a problemas), y se discute sobre las } \\
\text { propuestas. }\end{array}$ \\
\hline $\begin{array}{l}\text { Asociar } \\
\text { palabras }\end{array}$ & $\begin{array}{l}\text { Como preparación, se pide a los participantes que realicen } \\
\text { alguna actividad (buscar información, tomar fotos sobre el } \\
\text { tema, etc.) antes del desarrollo del grupo focal. }\end{array}$ \\
\hline $\begin{array}{l}\text { Probar un } \\
\text { producto }\end{array}$ & $\begin{array}{l}\text { Se visualian palabras libremente a un tema o a una palabra } \\
\text { incompletas propuestas por el moderador } \\
\text { cualquiera que sea el producto objeto de estudio, y se discute } \\
\text { sobre las opiniones que genera. }\end{array}$ \\
\hline
\end{tabular}

\section{Adaptación de la técnica de grupos focales al aula de lengua extranjera}

\subsection{Grupos focales y competencia comunicativa intercultural}

En el caso de estudiantes de lengua extranjera en su país de origen y en clases homogéneas, no es posible el acceso a los grupos de la cultura extranjera que requieren los proyectos etnográficos. Por otro lado, los alumnos constituyen un grupo que, con la aplicación de la técnica de grupos focales, puede convertirse en objeto de estudio con el fin de conocer con más profundidad la cultura y la sociedad a la que pertenecen. A través de los temas de investigación, se puede conseguir información y reflexionar sobre los estilos de vida de los alumnos, sus relaciones sociales, sus actitudes respecto de cualquier tema, y sobre la visión que los alumnos poseen de la propia y 
otras culturas. Todo ello significa que los alumnos van a adquirir conocimientos sobre cómo son ellos mismos y qué diferencias y similitudes socioculturales, y personales, existen entre ellos. Este incremento en los conocimientos sobre la propia cultura y sociedad, y sobre las similitudes y diferencias entre los alumnos, promoverá también una actitud de tolerancia hacia las diferencias y de curiosidad para conocer a los otros.

Para descubrir esos conocimientos, los alumnos van a realizar sus investigaciones, dirigir las discusiones y participar en las mismas. Todo ello estimulará las habilidades de búsqueda y descubrimiento, y las actitudes para ponerse en el lugar del otro e intentar comprender sus motivos. Por otro lado, las habilidades de interpretación y relación, y de interacción con miembros de otras culturas, son más difíciles de tratar en un grupo homogéneo. Además, las discusiones en los grupos focales promoverán la reflexión de los alumnos sobre su propia conducta y sus opiniones, reflexión que puede llevar también a la adquisición de una conciencia cultural crítica.

Finalmente, además de la competencia intercultural, las competencias de la lengua también serán promovidas con la aplicación de la técnica de grupos focales, ya que las preguntas, las discusiones y el informe se realizarán en la lengua meta.

\subsection{Grupos focales y actividades de expresión oral}

La aplicación en el aula de LE de la técnica de grupos focales presenta similitudes y diferencias con otros tipos de actividades de expresión oral, en concreto con los debates y las conversaciones. En cuanto a los debates, tanto estos como los grupos focales son discusiones dirigidas por un moderador y la conversación versa sobre un tema fijado de antemano. Por ello, en ambas técnicas se debe proporcionar a los alumnos recursos lingüísticos para preguntar y expresar opiniones, acuerdo y desacuerdo, para tomar, mantener y ceder el turno de palabra, para pedir aclaraciones, expresar juicios de valor y emociones, etc. Pero estas técnicas también presentan grandes diferencias. Una diferencia es el objetivo que se atribuye a la comunicación en cada técnica. En el debate, el objetivo de la comunicación es la "exposición y defensa de ideas frente o en apoyo de las ideas de otros interlocutores" (Pinilla 
2004, p. 893); pero en la discusión de un grupo focal, el objetivo de la comunicación es compartir la máxima información posible sobre la conducta, las opiniones y los sentimientos de los participantes que sea relevante para resolver el problema de investigación planteado. Además, el tipo de temas tratados en un debate y en un grupo focal también difiere. Mientras que en los debates se puede tratar cualquier tema que implique opiniones encontradas, los temas tratados en los grupos focales son sobre situaciones que los alumnos han vivido. Por ello, mientras que en el debate los alumnos deben documentarse sobre un tema y argumentar racionalmente sobre el mismo, en una discusión de grupo focal los alumnos deben realizar introspección para poder contar sus experiencias y las opiniones y emociones referentes a las mismas.

En cuanto a las conversaciones en el aula de LE, tanto en ellas como en las discusiones de los grupos focales los alumnos exponen sus opiniones respecto de un tema, pero en las conversaciones las intervenciones son más libres y están menos guiadas. Por otro lado, podemos distinguir entre dos tipos de conversaciones. El primero son las minicharlas, que consisten en la exposición de un tema por parte de un alumno, sobre el que los demás alumnos hacen preguntas y comentarios (Alonso, 1994). El segundo tipo son las conversaciones informales, que son conversaciones libres, que pueden saltar de un tema a otro libremente (Pinilla 2004). Mientras en las minicharlas la presentación del tema por parte de un alumno tiene más importancia que en los grupos focales, en las conversaciones informales los temas que se tratan a lo largo de las mismas aparecen de forma más espontánea que en los grupos focales, donde el tema está fijado de antemano y la discusión se desarrolla guiada por las preguntas del moderador.

\subsection{Técnica de grupos focales y programación del curso}

Como los alumnos van a realizar una pequeña investigación social, es necesario dedicar algunas sesiones a su formación metodológica para aplicar la técnica de grupos focales. No se trata de crear investigadores sociales, sino de que adquieran un cierto dominio de técnicas de investigación que permiten profundizar en el conocimiento sociocultural, las cuales una vez adquiridas 
podrán ser aplicadas de manera más informal por el alumno a lo largo de su vida para adquirir nuevos conocimientos.

La necesidad de incluir sesiones preparatorias, y distribuir la realización de los grupos focales a lo largo de diferentes sesiones, hace necesario que esta técnica no sea aplicada de forma puntual en un momento dado del curso, sino que el curso esté programado para realizar investigaciones con la técnica de grupos focales en el aula a lo largo del mismo. Las primeras sesiones deben dedicarse a la formación de los alumnos para la elaboración del problema de investigación, la formulación de preguntas adecuadas, la elaboración de la guía de discusión, la adquisición de estrategias para dirigir la discusión, el aprendizaje de la forma de análisis de los datos y de la elaboración del informe de conclusiones. En las siguientes sesiones, se desarrollarán grupos focales dedicados cada vez a un tema concreto. Se trata de que a lo largo del curso todos los alumnos puedan realizar el rol de investigador, y que en el resto de sesiones realicen el rol de participantes en el grupo de discusión. Además, es necesario proporcionar a los alumnos recursos comunicativos para que puedan desarrollar las discusiones. Por ello, el profesor deberá tomar una serie de decisiones sobre la organización del curso:

1. Número de sesiones que va a dedicar a la formación de los alumnos en la técnica de grupos focales, y qué actividades y materiales va a usar para ello.

2. Número de alumnos que realizarán el rol de investigador (moderador y observador) en cada sesión. Se trata de que todos los alumnos realicen una investigación, individualmente o en pequeños grupos, y por lo tanto el número de grupos y de miembros dependerá del número de alumnos en la clase.

3. Qué temas van a ser objeto de investigación y cómo apoyar a los estudiantes para que formulen problemas de investigación sobre los mismos.

4. Forma de supervisión de la guía de discusión. Ya que el diseño de una buena guía es fundamental para el buen desarrollo de esta técnica, el profesor deberá supervisar y apoyar la elaboración de la guía de cada 
alumno.

5. Temporización de las diferentes actividades. Ya que los alumnos van a presentar el informe de conclusiones en la clase y se van a intercambiar impresiones sobre el mismo, es necesario reservar un tiempo de la clase para el mismo. El profesor deberá decidir cómo distribuye el tiempo de la clase para tareas de preparación, realización de los grupos focales, y presentación de los resultados.

6. Qué recursos comunicativos necesitarán los alumnos para desarrollar el grupo focal sobre cada tema de investigación, y qué actividades van a realizarse y con qué materiales para adquirir dichos recursos.

\subsection{Un ejemplo de aplicación de grupos focales en el aula de lengua extranjera}

Hemos realizado de forma experimetal una actividad de grupos focales en una clase de ELE de 12 alumnos de nivel A2+ de una universidad japonesa. Seguidamente la exponemos, mostrando a partir de esta experiencia las distintas fases de las que consta la aplicación de los grupos focales en el aula de LE. A diferencia de una investigación real, en el aula de LE no es necesario seleccionar a los participantes en el grupo focal, ya que son todos los alumnos de la clase." Así, el número de fases de la aplicación de esta técnica en el aula de LE queda reducido a seis (Figura 1):

1. Definición del problema de investigación. Los temas de investigación elegibles pueden ser proporcionados por el profesor, lo cual da unidad al curso y orienta a los alumnos. En nuestro caso, elegimos el tema de los hábitos alimenticios.

2. Formulación del problema de investigación. El problema concreto de investigación debe ser elaborado por los alumnos que realizan el rol de investigadores en cada clase, ya que así se ven obligados a reflexionar sobre los temas y pueden adaptarlos a sus propios intereses. En nuestra experiencia elegimos el problema: “¿Los hábitos alimenticios de los jóvenes japoneses son sanos?"

3. Elaboración de la guía de discusión. Los alumnos-investigadores 
deben elaborar la guía de acuerdo con los requisitos que hemos establecido en las secciones anteriores. En nuestro caso, después de comentar una lectura sobre la comida rápida y la alimentación equilibrada, entre todos los alumnos prepararon la guía de discusión (véase la Tabla 3) a partir de una lluvia de ideas.

4. Desarrollo de la sesión de grupo focal. En la siguiente clase, el profesor realizó el papel de moderador del grupo focal y varios estudiantes se fueron relevando como observadores.

5. Análisis e interpretación de los datos. El análisis de datos debe explicar, en referencia al problema de investigación, qué conductas, opiniones y experiencias expresan los alumnos, qué sentimientos asocian a las mismas, qué motivos atribuyen a las mismas, y qué opinan sobre las respuestas de los otros alumnos. En nuestra experiencia, el profesor realizó un resumen de la discusión, intentando encontrar patrones de conducta entre las respuestas y los motivos de las diferentes conductas. Se concluyó que en la clase había dos grupos de alumnos según sus hábitos alimenticios. Uno de ellos mantenia hábitos bastante saludables, mientras que el otro grupo tenía unos horarios muy desordenados, tomaba mucha comida rápida y cocinaba básicamente frituras. El principal motivo que determinaba los hábitos de los alumnos era estar ocupado o no con un trabajo a tiempo parcial. Además, en general los alumnos no pensaban mucho sobre la salud, y

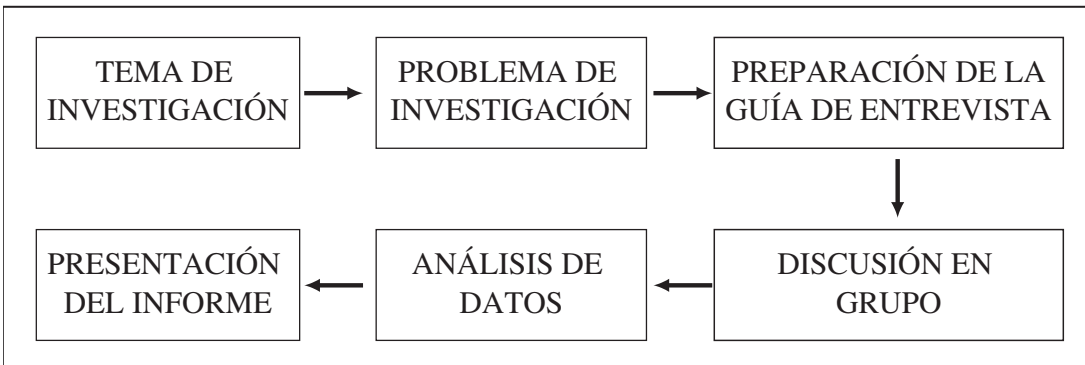

Figura 1: Fases de una investigación con grupos focales en el aula de lengua extranjera. 
cuando vivían solos abandonaban el desayuno típico japonés.

6. Elaboración del informe de conclusiones. En la tercera clase se presentó a los alumnos el informe, conteniendo un resumen de la discusión y las conclusiones.

Tabla 3: Guía de discusión sobre los hábitos alimenticios

\begin{tabular}{|c|c|}
\hline Introducción & $\begin{array}{l}\text { Hoy vamos a discutir sobre vuestros hábitos alimenticios para } \\
\text { conocer si los hábitos alimenticios de los estudiantes de esta } \\
\text { universidad son sanos o no. }\end{array}$ \\
\hline $\begin{array}{l}\text { Tema 1: } \\
\text { Los hábitos } \\
\text { diarios } \\
\text { (15 minutos) }\end{array}$ & $\begin{array}{l}\text { 1. Contadme cómo son vuestras comidas diarias. } \\
\text { ¿Cuántas veces coméis al día? } \\
\text { ¿A qué hora desayunáis / coméis / cenáis? } \\
\text { 2. ¿Cuánto suele durar cada comida? } \\
\text { 3. ¿Soléis tomar algo entre comidas? } \\
\text { ¿Qué? ¿Cuándo? } \\
\text { ¿Con qué frecuencia? } \\
\text { 4. ¿Con quién soléis tomar las diferentes comidas del día? }\end{array}$ \\
\hline $\begin{array}{l}\text { Tema } 2 \text { : } \\
\text { Las comidas } \\
(30 \text { minutos })\end{array}$ & $\begin{array}{l}\text { 5. ¿Qué soléis tomar en las distintas comidas del día? } \\
\text { ¿Con qué frecuencia coméis fruta y verdura? } \\
\text { ¿Con qué frecuencia coméis carne? ¿De qué tipo? } \\
\text { ¿Con qué frecuencia coméis pescado? } \\
\text { 6. ¿Con qué frecuencia tomáis alimentos precocinados? } \\
\text { ¿Cuáles? ¿En qué situaciones? } \\
\text { 7. ¿Dónde soléis tomar las distintas comidas del día? } \\
\text { ¿Con qué frecuencia coméis fuera de casa? ¿Dónde? } \\
\text { ¿Quién cocina en vuestra casa? } \\
\text { 8. ¿Qué opináis sobre la “comida rápida”? } \\
\text { ¿Con qué frecuencia tomáis "comida rápida”? } \\
\text { ¿Cuándo la tomáis? ¿Con quién? }\end{array}$ \\
\hline $\begin{array}{l}\text { Tema 3: } \\
\text { Cuidado de } \\
\text { la salud } \\
\text { (15 minutos) }\end{array}$ & $\begin{array}{l}\text { 9. ¿Qué hacéis para cuidar vuestra salud? } \\
\text { 10. ¿Qué pensáis de las dietas? } \\
\text { ¿Qué tipos de dieta conocéis? } \\
\text { ¿Has pensado en hacer dieta alguna vez? }\end{array}$ \\
\hline & 11. Esto es todo. ¿Queréis decir algo más? \\
\hline
\end{tabular}




\section{Conclusiones}

El objetivo del presente artículo ha sido establecer una metodología para la enseñanza/aprendizaje de la competencia comunicativa intercultural en el aula de LE. Hemos diseñado el método pensando en hacer frente al problema de la falta de acceso a otras culturas que se produce cuando los alumnos estudian en su país de origen.

Para diseñar nuestra metodología nos hemos basado en el concepto de competencia comunicativa intercultural establecido por Byram (1997). A partir de las aportaciones de Byram hemos definido los objetivos de las actividades de los alumnos, que son promover actitudes de curiosidad, empatía y respeto hacia otras culturas, promover habilidades para descubrir e interpretar elementos culturales, adquirir conocimientos sobre la propia y otras culturas, y desarrollar una conciencia crítica para evaluar racionalmente elementos culturales.

Para desarrollar nuestro método hemos adaptado al aula de LE la técnica de investigación social de grupos focales. Con este procedimiento, los estudiantes son a la vez investigadores de su cultura y objeto de la investigación. Los alumnos tienen que preparar un problema de investigación sobre las experiencias, las opiniones y los sentimientos de sus compañeros sobre diversos temas de su propia vida, a partir de los cuales puedan conocer mejor la propia cultura y sociedad, y las diferencias intraculturales. Con ello, además de incrementar los conocimientos sobre su cultura y situarse en una posición mejor para compararla con otras culturas, los alumnos aumentarán sus habilidades para descubrir aspectos socioculturales, su capacidad de introspección, su conciencia intercultural, sus actitudes de curiosidad, empatía y tolerancia respecto de opiniones diferentes a las suyas, y su competencia comunicativa, ya que adquirirán recursos comunicativos para realizar las discusiones en grupo en la LE.

En definitiva, la aplicación de la técnica de grupos focales en las clases de LE nos ofrece un procedimiento sistemático para organizar las clases orientadas a la interculturalidad, y nos orienta en el diseño de materiales para apoyar a los alumnos en el aprendizaje de la técnica y de recursos comunicativos. El desarrollo de materiales para el aprendizaje de la técnica de 
grupos focales, y la aplicación práctica de este nuevo método, son los próximos temas que deben ser investigados.

\section{Notas}

1) Byram (1997) utiliza los términos en francés: savoir être, savoirs, savoir comprendre, savoir apprendre/faire, y savoir s'engager.

2) Las aportaciones a la aplicación de la técnica etnográfica en la enseñanza de LE más destacadas son Oullet, 1984; Byram y Esarte-Sarries, 1991; Barro et al., 1998; Byram y Fleming, 1998; y Roberts et al., 2001.

3) Para que las preguntas estimulen la discusión se propone que sean del tipo “¡cómo...?” o “¿en qué situación...?”. Por ejemplo, en un grupo focal sobre un anuncio publicitario son habituales las preguntas del tipo: “¿cómo te sentiste al ver al personaje que usa el producto X?" (Krueger y Casey, 2000).

4) Por ejemplo, analizando las actitudes sobre personas de otras razas, se pueden presentar fotos de personas de distantas razas y pedir a los participantes en la discusión que elijan la persona que preferirían que fuera su compañero de trabajo, su médico, la persona con la que se casara un familiar próximo, etc. (Krueger et al., 2000).

5) Si la clase pertenece a una institución de educación reglada, se convierte en representativa de una determinada población, por ejemplo: los estudiantes universitarios japoneses del tercer curso de carrera (con 20 o 21 años). Ello hace que constituyan un buen grupo para analizar las actitudes y conductas de los estudiantes de un país.

\section{Referencias}

Alonso, E., 1994. ¿Cómo ser profesor/a y querer seguir siéndolo? Madrid: Edelsa.

Barro, A., Jordan, S., Roberts, C., 1998. "Cultural practice in everyday life: the language learner as ethnographer". En: Byram, M. y Fleming, M. eds. 1998, pp. 76-97.

Byram, M., 1997. Teaching and Assessing Intercultural Communicative Competence. Clevedon, Philadelphia, Toronto, Sydney, Johanesburg: Multilingual Matters.

Byram, M. y Esarte-Sarries, V., 1991. Investigating Cultural Studies in Foreign Language Teaching. Clevendon, Philadelphia: Multilingual Matters.

Byram, M. y Fleming, M. eds. 1998. Language Learning in Intercultural Perspective: Approaches Through Drama and Ethnography. Cambridge: Cambridge University Press. 
Byram, M., Nichols, A. y Stevens, D. eds., 2001. Developing Intercultural Competence in Practice. Clevedon, Buffalo, Toronto, Sydney: Multilingual Matters.

Byram, M., Nichols, A. y Stevens, D. eds., 2003. Intercultural Experience and Education. Clevedon, Buffalo, Toronto, Sydney: Multilingual Matters.

Corbett, J., 2003. An Intercultural Approach to English Language Teaching. Clevedon, Buffalo, Toronto, Sydney: Multilingual Matters.

Instituto Cervantes, 2007. Plan Curricular del Instituto Cervantes. Niveles de referencia para el Español B1/B2. Madrid: Insituto Cervantes, Biblioteca Nueva.

Krueger, R.A. y Casey, M.A., 2000. Focus Gropus. A Practical Guide for Applied Research. Thousand Oaks, London, New Delhi: SAGE Publications.

Morgan, D.L., 1997. Focus Groups as Qualitative Research. Thousand Oaks, London, New Delhi: SAGE Publications.

Oullet, F., 1984. « Éducation, compréhension et communication interculturelles: essai de clarification des concepts ». Éducation Permanente, n. 75.

Pinilla, R., 2004. "La expresión oral”. En: Sánchez-Lobato, J. y Santos-Gargallo, I. eds., 2004, pp. 879-897.

Roberts, C., Byram, M., Barro, A., Jordan, S., Street, B., 2001. Language Learners as Ethnographers. Clevedon: Multilingual Matters.

Sánchez-Lobato, J. y Santos-Gargallo, I. eds., 2004. Vademécum para la formación de profesores. Enseñar español como segunda lengua (LE) / Lengua extranjera (LE). Madrid, SGEL.

Sercu, L., Bandura, E., Castro, p. , Davcheva, L., Laskaridou, Ch., Lundgren, U., Méndez, M., Ryan, p. 2005. Foreign Language Teachers and Intercultural Competence. Clevedon, Buffalo, Toronto: Multilingual Matters.

Stewart, D.W., Shamdasani, p. N. y Rook, D.W., 2007. Focus Groups. Theory and Practice. Thousand Oaks, London, New Delhi: SAGE Publications. 


\section{La técnica de grupos focales como método para el desarrollo de la competencia comunicativa intercultural}

\section{Lluís Valls Campà}

En el artículo se presenta una adaptación de la técnica de investigación social de grupos focales como método didáctico para promover el desarrollo de la competencia comunicativa intercultural de los alumnos de ELE. Con ello se prentende corregir algunos problemas que plantea la aplicación didáctica del método etnográfico. Aplicando la técnica de grupos focales, los alumnos plantean problemas de investigación sociocultural y realizan discusiones en clase para resolverlos. Con este proceso se promueven las habilidades y actitudes interculturales de los alumnos, sus conocimientos socioculturales y una conciencia cultural crítica, además de mejorar su competencia comunicativa en español. 